\title{
AAP Presidential Address The AAP and the transformation of medicine
}

\author{
Ralph Snyderman, M.D.
}

As much as I enjoy blazing new trails, my approach to this Presidential Address has followed the requisites faithfully taken by my illustrious predecessors. I have read the Constitution of the Association of American Physicians (AAP) developed in 1886, I have reviewed the history of the organization, and I have studied most of the Presidential Addresses delivered over the last decade.

Sometime in the mid 1970s, I recall sitting in the audience listening to the Presidential Address delivered by Hollie Smith. My thoughts at that time were transfixed on his brilliance, wit, and the glory of being the President of the AAP. Nonetheless, all things considered, I concluded I'd rather be in my mid-thirties and sitting in the audience. Little did I ever imagine that someday I would be addressing this august body with some thirty-something thinking the same thing about me.

In 1886, the AAP defined its goals as the advancement of scientific and practical medicine, the development of a Society where like-minded physicians could discuss their work, and the creation of an association for colleagues who have made substantial contributions to the body of medical knowledge (Table 1).

During the last two decades, our organization has undergone substantial change, and the introspection of our Presidents' thinking about the contemporary role of the AAP is evident from the topics they presented. The dominant focus of recent Presidential Addresses has been oriented towards quo vadis - where is the AAP now and where should it be going? Many have wondered if it is indeed a viable organization. Most talks have focused on how much better things were during the good old days, the plight of falling memberships, and the risk for physician-scientists - particularly clinical researchers - to become extinct. During his Presidential Address in 2001, Dr. Robert Lefkowitz showed a chart (Figure 1)

This article is adapted from a presentation at the ASCI/AAP Joint Meeting, April 16-18, 2004, in Chicago, Illinois, USA.

Citation for this article: $J$. Clin. Invest. 114:1169-1173 (2004). doi:10.1172/JCI200423463.

Table 1

The goals of the AAP (1886)

"The advancement of scientific and practical medicine" (Constitution 1886)

"A society.... in which we could meet our fellows in the same line of work" (Osler)

"We want an association composed of members, each one of whom is able to contribute something real to the common stock of knowledge, and where he who reads such a contribution feels sure of a discriminating audience. ... We also want a society in which we can learn something"

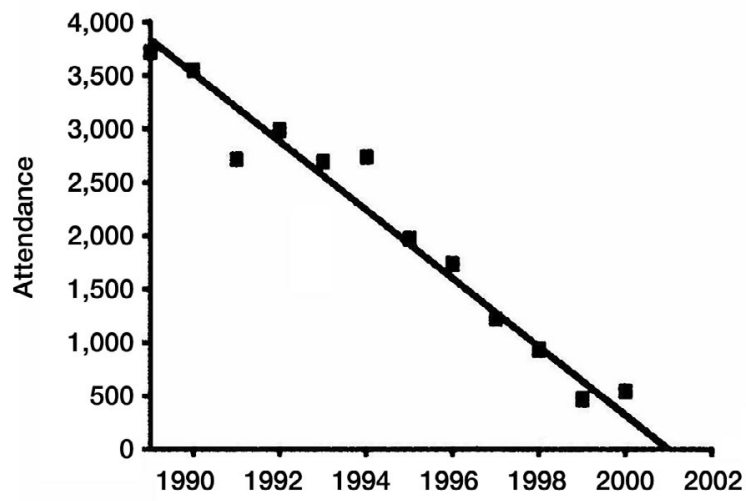

Figure 1

Total attendance at recent annual spring meetings.

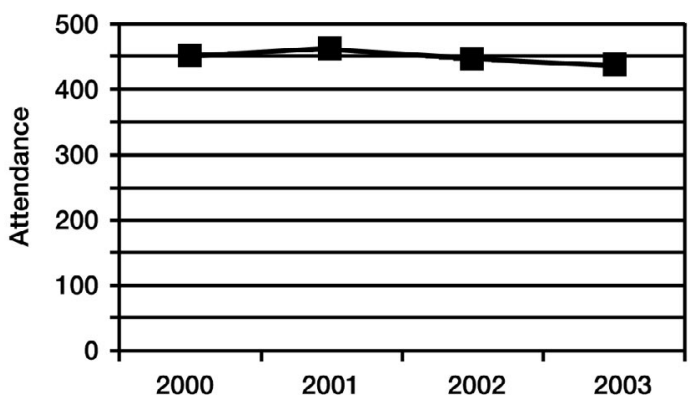

Figure 2

Attendance at recent ASCI/AAP meetings.

describing the fall in attendance at our annual spring meetings, with a regression line projecting no attendance by 2001 (1).

As anticipated, this did not prove to be the case (Figure 2). I suspect that the stable membership of the AAP and ASCI will likely cause attendance to plateau and continue until the mortality rate of members substantially exceeds the recruitment of new members. Nonetheless, the vitality of our organization, as measured by interest in membership and attendance at national meetings, is status quo at best.

Like many AAP Presidents before me, I believe this is a critical time in the life of our association and a time for considering change. As the esteemed late twentiethcentury athlete-philosopher Yogi Berra said, "When you come to a fork in the road - take it." 


\section{supplement}

More importantly, as Brutus said to Cassius in Shakespeare's Julius Caesar:

There is a tide in the affairs of men, Which taken at the flood, leads on to fortune;

Omitted, all the voyage of their life

Is bound in shallows and in miseries.

On such a full sea are we now afloat,

And we must take the current when it serves,

Or lose our ventures.

I believe that we are in such a time in the life of the AAP, and we have an opportunity, if not an obligation, to refocus and energize our association. I believe we must dedicate ourselves to use its influence for the good of physician-directed biomedical research leading to improvement in the health of all.

We are on the verge of a great transformation in medicine, one at least as great in scope as that faced by the founders of the AAP. As shown in Figure 3, the profession of medicine is noted in the earliest recorded historical documents, including the Papyrus papers of approximately 3500 BCE. Nonetheless, the practice was not substantially influenced by the emergence of science until the latter part of the 1800 s. In my view, it is not by accident that the AAP held its first meeting in 1886. This was a time when the practice of medicine was just beginning to undergo dramatic changes due to the emergence of scientific know-how. At the time of the first meetings of the AAP, emerging sciences of anatomy, chemistry, germ theory, and physics had the potential to revolutionize the practice of medicine (Figure 4). Nonetheless, in the United States the practice was unscientific, anecdotal, and unregulated with MD degrees being given by more than 800 storefront medical schools.

The giants of medicine who formed the AAP were associated with the small number of academic medical centers dedicated to the incorporation of science into the practice of medicine. In the early 1900s, a report commissioned by the Carnegie Foundation, termed the Flexner Report (2), recognized a tremendous discrepancy between medical education and practice and scientific capabilities.

I would argue that the membership of the AAP had a tremendous amount to do with the transformation of medicine at that time, from anecdotal and unscientific to a model embraced by the contemporary academic medical center; that is, a medical school associated with a teaching hospital and a faculty

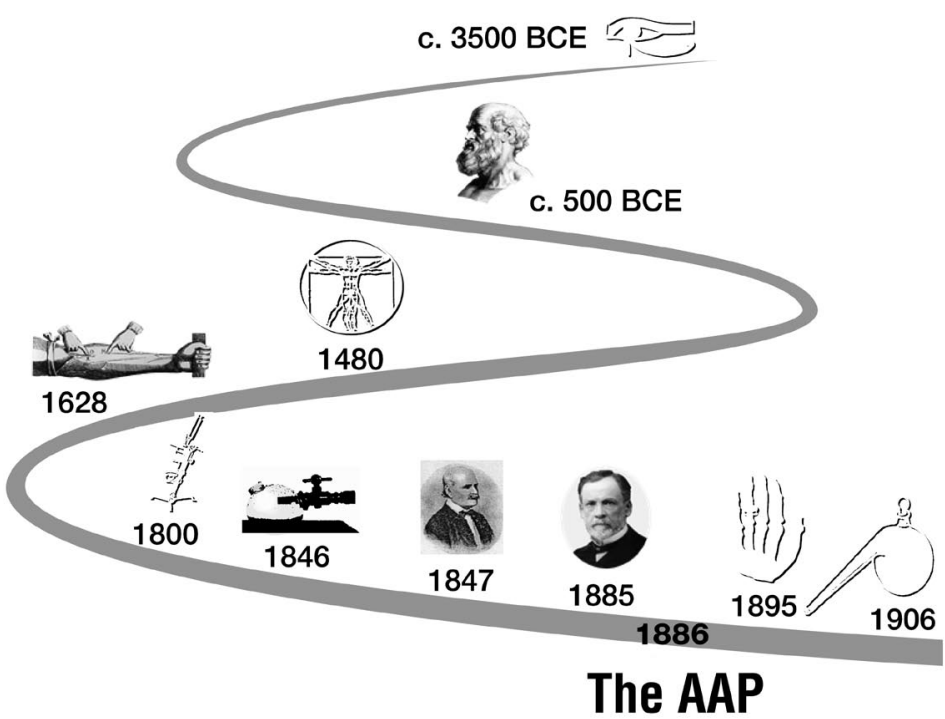

Figure 3

History of medicine over the ages.

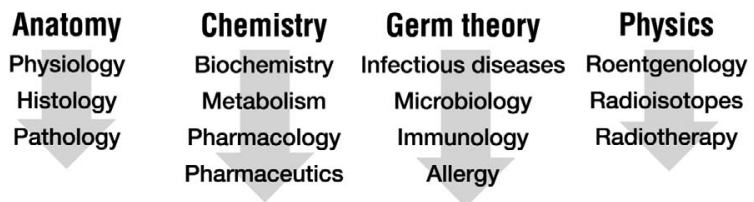

Figure 4

Factors impacting medicine in the 1890s.

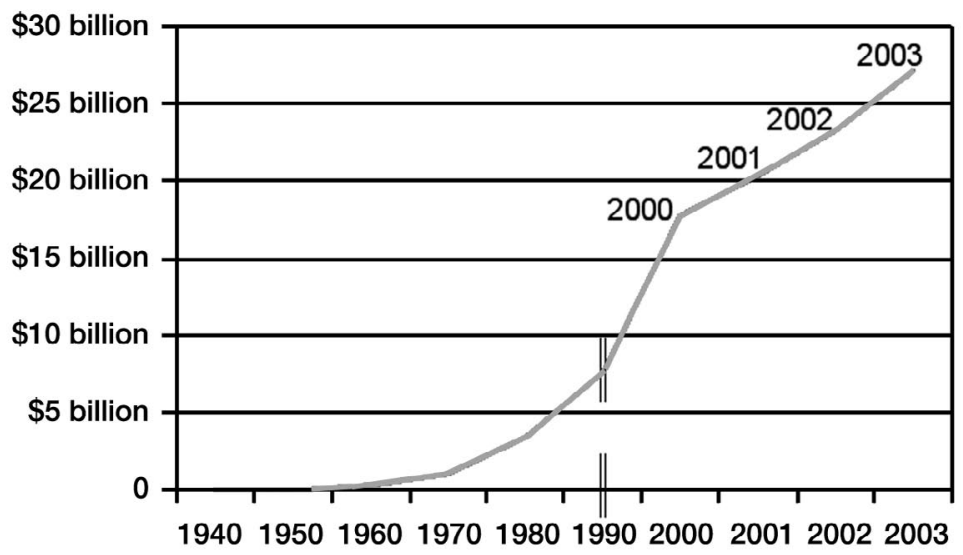

Figure 5

$\mathrm{NIH}$ appropriations.

heavily engaged in research. This model led to an explosion in our understanding of the pathophysiological basis of disease.

Supported by wise investments on the part of our government following WWII, through leadership of Vanivear Bush, the presidential scientific advisor who helped develop the Manhattan Project, there have been robust federal investments in the biomedical sciences.
Appropriations to the National Institutes of Health increased markedly in the 1960s and continued to rise steadily until the year 2000, when the NIH budget began to be doubled over five years (Figure 5). This investment on the part of the federal government as well as by industry has led to another explosion in scientific capabilities, and we are just beginning to sense the magnitude of its effects. 
Table 3

Genomics and health

Therapeutic proteins

Therapeutic targets

Pharmacogenetics

Gene therapy

> SUSCEPTIBILITY PROFILES

Table 4

Risk profile analysis

Alzheimer disease

Bipolar disorder

Colon cancer

Coronary artery disease

Diabetes

Epilepsy

Hypertension

Ovarian cancer

Parkinson disease

Prostate cancer

Schizophrenia

\section{Table 2 \\ Factors impacting medicine in 2004 \\ Genomics \\ Proteomics \\ Metabolomics \\ Medical technologies \\ Informatics}

Breast cancer

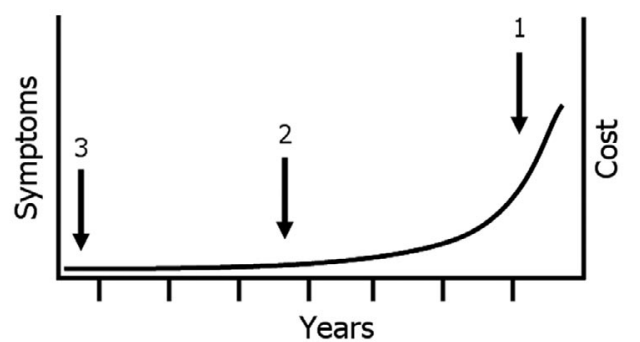

\section{Figure 6}

Disease progression (\#1 current practice, \#2 current capability, \#3 future capability).

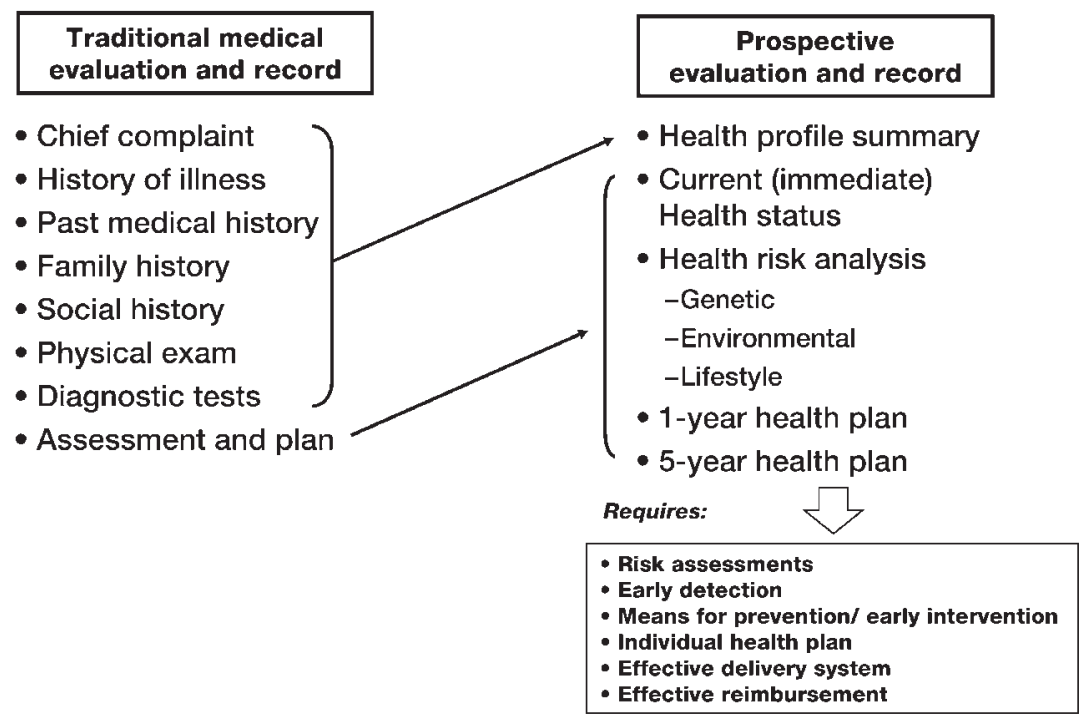

Figure 7

Paradigm shift.

The fields of genomics, proteomics, metabolomics, medical technologies, and informatics have provided us with knowledge and discoveries that could not have been anticipated even a decade ago (Table 2 ). When one looks at the impacts of genomics alone, one can envision a plethora of new therapeutic proteins, a better definition of therapeutic targets, the emerging field of pharmacogenetics, and the eventual success of gene therapy. But, I would argue that amongst the most powerful new capabilities emerging, in part from genetics, is the ability to determine an individual's risk for development of disease. The importance of this capability for the practice of medicine is all the more important when contrasted with the current way that medicine is practiced (Table 3 ).
Chronic diseases account for two-thirds of all health-care expenditures and are an increasing burden to our society. Currently, our health-care delivery system focuses on the far right-hand side of the disease progression curve long after the initial onset of disease (Figure 6, point 1 ), and often for acute episodes of largely irreversible processes. Even today, the practice of medicine, if taking full advantage of scientific capabilities, could move the curve far to the left at the earliest incipient events associated with chronic disease (Figure 6, point 2). If one then envisions the power of genomic information, the curve can be shifted even further to the left (Figure 6, point 3), allowing a risk analysis for most important complex diseases (3).
In querying geneticists about the likelihood of far better understanding of riskprofile analysis for common chronic diseases, I have assembled the list shown in Table 4. Information such as this could enable an approach to health care that is informed by an individual's genetic risk for developing serious diseases later in life. This would enable a health-care delivery approach that encompasses risk analysis long before diseases occur. Our current health-care system is in danger of collapse. It is unsustainable in its present form because of its expense, inefficiency, and unfairness.

I believe we need a paradigm shift from the current practice of medicine, seen on the left-hand side of Figure 7, which describes the current medical record and, through it, how medicine is practiced (4). 
The current medical record, starting with a chief complaint, is really a "root cause analysis of failure." We do this very well when patients come to us with a particular disease process. However, there is no business, nor should there be any individual's life, that does not have a strategic plan to prevent errors from happening, and if they do, to intervene at the earliest possible time. Every one of us is engaged in planning, and I doubt if anyone in this audience doesn't have a retirement plan. How many of us have a health plan that will assure, to the maximum degree possible, good health at the time that we retire? What we need is a health-care system that is prospective, that incorporates risk analysis, and that leads to personalized strategic health planning. This approach would not have been possible a decade ago but is increasingly approachable due to scientific advances and know-how.

This is the point of my talk - the current discrepancy between scientific know-how and the practice of medicine is greater today than at any time since the founding of the AAP. We are practicing disease-based medicine while research is giving us the ability to deliver prospective health care with personalized health planning. We need to understand and articulate the role of the physicianscientist in bringing this about as well as the power of prospective health care in improving our nation's health.

To develop personalized health planning, one needs constantly improving tools for personal health risk assessment leading to the development of a personalized health plan. In order to develop such a risk assessment tool, one needs to have highly refined clinical information, biomarkers of disease, genomic and other risk prediction, and molecular imaging again to evaluate molecular mechanisms at the tissue level. Information in all of these areas is dependent on a robust and focused research enterprise. Data and literature mining is absolutely dependent on clinical research. Outcomes tracking, development of pharmaceutical procedures, and other interventions again are dependent on a base of research (Figure 8) (3).

An advantage of prospective health is shown in Figure 9 (4). Risk-assessment tools and health-risk-assessment models allow the segmentation of populations into categories, which allows individuals to have access to the exact level of health that they need. One can also focus aggressively on modifying risk before disease occurs or to intervene at the earliest onset. I am proud to say that we are already implementing such a rudimentary healthcare plan at Duke.

The importance of this to the AAP is shown in Table 5. In order to effect a more rational health-care delivery system and promote health and wellness of all, one is absolutely dependent on research research, I argue, conducted by physicians. The full range of basic discovery research all the way through to classical epidemiology is requisite to developing personalized 


\section{Table 5}

Types of research needed

Basic discovery

- Non disease-oriented

- Disease-oriented

Translational research

Clinical trials

Outcomes research

Health policy

Classical epidemiology

medicine and to improving health care. We as the first and most senior organization representing the physician-scientist must make our voice heard to help rationalize our currently flawed health-care system. Leadership from physician-scientists must articulate the vision of a far better model of delivery. With such a model in mind, the value of research will be better understood and applied to solve problems. Such a vision along with implementation into clinical delivery models will allow politicians to understand better the need to fund the academic medical infrastructure. The role of the physician-scientist is paramount to progress in health care.

My esteemed colleague, mentor, and first academic medicine boss Jim Wyngaarden clearly identified "the clinical investigator as an endangered species" in 1979 (5). Since that time, the situation has continued to be threatening. Nonetheless, well-mean- ing institutions, particularly the NIH, have thrown their weight behind efforts to ensure the continued production of physician-scientists. Taking a business-like view of the health-care market, I believe that the physician-scientist will be increasingly valued as being mission critical for enabling prospective health care, but we must hasten this process and articulate our values in order to change our status as an endangered species to an emerging species (6). The implementation of the NIH Roadmap is a process to focus research towards the common good and physician-scientists should be leading the charge.

Models of prospective care that are emerging in primitive forms are already providing the template which not only treats disease better but also anticipates it and intervenes before it occurs. The fragility of our current health-care system, the need for a more rational cost effective system and the power of science to help bring this about make a compelling case for the need of physician-scientists playing their multiple roles in applying discovery to practice.

I therefore conclude that now, similar to 1886 , is a threshold opportunity for AAP. Over a century ago, the AAP helped bring science to medicine so it could treat disease. Now it can lend its voice to articulating the power of science to transform health-care delivery to a model of personalized disease prevention.

During its previous years, our organization has served the role envisioned by the founders: meet, learn, honor, and mentor. We should do this, but we must do more. I suggest we move consciously and forcefully to represent and articulate the power of the physician-scientist in transforming health care. We must better understand our role in this and support change in how medicine is practiced. Our forefathers did just this by introducing science into medicine. We must now utilize the emerging new sciences to transform health care to anticipate, prevent, and minimize disease. Recognizing our role in promoting solutions, based on good science, is what we can and should do well.

Address correspondence to: Ralph Snyderman, Chancellor Emeritus, Duke University, Box 3059, Durham, North Carolina 27710, USA. Phone: (919) 6842345: Fax: (919) 681-9977; E-mail: ralph. snyderman@duke.edu.

1. Lefkowitz, R.J. 2001. 2001: An AAP Odyssey. [Presidential Address, Association of American Physicians.] J. Clin. Invest. 108:s9-s13.

2. Flexner, A. Medical Education in the United States and Canada: A report to the Carnegie Foundation for the Advancement of Teaching. Bulletin No. 4. Boston: Updyke, 1910.

3. Snyderman, R., and Williams, R.S. 2004. Prospective medicine: the next health care transformation. Acad. Med. 78:1079-1084.

4. Langheier, J., and Snyderman, R. 2004. Prospective medicine: the role for genomics in personalized health planning. Pharmacogenomics. 5:1-8.

5. Wyngaarden, J.B. 1979. The clinical investigator as an endangered species. N. Engl. J. Med. 301:1254-1259.

6. Snyderman, R. 2004. The Clinical researcher - an "emerging" species. JAMA. 291:882-883. 